\title{
The burden of brain hypoxia and optimal mean arterial pressure in patients with hypoxic ischemic brain injury after cardiac arrest
}

\author{
Mypinder S Sekhon MD ${ }^{1}$, Peter Gooderham $\mathrm{MD}^{2}$, David K Menon MD PhD 3 , \\ Penelope M A Brasher $\mathrm{PhD}^{4}$, Denise Foster $\mathrm{RN}^{1}$, Danilo Cardim $\mathrm{PhD}^{5}$, Marek Czosnyka $\mathrm{PhD}^{3}$, \\ Peter Smielewski $\mathrm{PhD}^{3}$, Arun K Gupta MBBS $\mathrm{PhD}^{3}$, Philip N Ainslie $\mathrm{PhD}^{6}$ and \\ Donald E G Griesdale MD MPH ${ }^{1,4,5}$
}

${ }^{1}$ Division of Critical Care Medicine, Department of Medicine, Vancouver General Hospital, West 12th Avenue, University of British Columbia, Vancouver, BC, Canada, V5Z 1M9.

${ }^{2}$ Division of Neurosurgery, Department of Surgery, Vancouver General Hospital, West 12th Avenue, University of British Columbia, Vancouver, BC, Canada, V5Z 1M9.

${ }^{3}$ Department of Clinical Neurosciences, Addenbrookes Hospital, University of Cambridge. Cambridge, United Kingdom

${ }^{4}$ Centre for Clinical Epidemiology and Evaluation, Vancouver Coastal Health Research Institute, 899 West 12th Avenue, University of British Columbia, Vancouver, BC, Canada, V5Z 1M9 ${ }^{5}$ Department of Anesthesiology, Pharmacology and Therapeutics, Vancouver General Hospital, West 12th Avenue, University of British Columbia, Vancouver, BC, Canada, V5Z 1M9. ${ }^{6}$ Department of Health and Exercise Sciences, University of British Columbia - Okanagan. Kelowna, BC, Canada

Corresponding Author:

Dr. Mypinder S. Sekhon

Critical Care Medicine

Vancouver General Hospital

Phone: 604-875-5949, Fax: 604-875-5957, Email: mypindersekhon@gmail.com

Address for reprints \& reprints will be ordered:

Room 2438, Jim Pattison Pavilion, $2^{\text {nd }}$ Floor, 855 West $12^{\text {th }}$ Avenue, Vancouver BC V5Z 1M9

Running title: Optimal mean arterial pressure and brain oxygenation post cardiac arrest

Conflicts of Interest and Source of Funding: PS and MC have financial interest in licensing ICM+ software (Cambridge Enterprise Ltd). The remaining authors have disclosed they have no conflicts of interest. This study was funded by an operating grant from the Laerdal Foundation. DG is funded through a Health-Professional Investigator Award from the Michael Smith Foundation for Health Research.

Keywords: Hypoxic ischemic brain injury, brain tissue oxygenation, cardiac arrest, optimal mean arterial pressure, autoregulation, pressure reactivity index

Word Count: 4529 


\begin{abstract}
Objectives: In patients at risk of hypoxic ischemic brain injury following cardiac arrest, we sought to (i) characterize brain oxygenation and determine the prevalence of brain hypoxia, (ii) characterize autoregulation using the pressure reactivity index (PRx) and identify the optimal mean arterial pressure (МАР ${ }_{\mathrm{OPT}}$ ), and (iii) assess the relationship between MAP tissue oxygenation $\left(\mathrm{PbtO}_{2}\right)$.
\end{abstract}

Design: Prospective interventional study.

Setting: Quaternary intensive care unit.

Patients: Adult patients with return to spontaneous circulation (ROSC) greater than 10 minutes and a post-resuscitation Glasgow Coma Score under 9 within 72 hours of cardiac arrest.

Interventions: All patients underwent multimodal neuromonitoring which included: (i) $\mathrm{PbtO}_{2}$, (ii) intracranial pressure; (iii) jugular venous continuous oximetry $\left(\mathrm{SjvO}_{2}\right)$; (iv) regional saturation of oxygen $\left(\mathrm{rSO}_{2}\right)$ using near-infrared spectroscopy, and (iv) PRx based determination of MAP ${ }_{\mathrm{OPT}}$, lower and upper limit of autoregulation. We additionally collected MAP, end tidal carbon dioxide $\left(\mathrm{ETCO}_{2}\right)$ and temperature. All data were captured at $300 \mathrm{~Hz}$ using $\mathrm{ICM}+{ }^{\circledR}$ brain monitoring software.

Measurements and Main Results: Ten patients ( 7 males) were included with a median age 47 (range $20-71)$ and ROSC 22 minutes $(12-36)$. The median duration of monitoring was 47 hours (15 - 88) and median duration from cardiac arrest to inclusion was 15 hours (6 - 44). The mean $\mathrm{PbtO}_{2}$ was $23 \mathrm{mmHg}$ (SD 8) and the mean percentage of time with a $\mathrm{PbtO}_{2}$ below 20 $\mathrm{mmHg}$ was $38 \%(6-100)$. The mean PRx was $0.23(0.27)$ and the percentage of time with a PRx greater than 0.3 was $50 \%(12-91)$. The mean MAP were $89 \mathrm{mmHg}(11), 82 \mathrm{mmHg}$ (8) and $96 \mathrm{mmHg}$ (9), respectively. There was marked betweenpatient variability in the relationship between MAP and indices of brain oxygenation. As the patients' actual MAP approached MAPOPT, $\mathrm{PbtO}_{2}$ increased $(\mathrm{p}<0.001)$. This positive relationship did not persist when the actual MAP was above MAPOPT.

Conclusions: Episodes of brain hypoxia in HIBI are frequent and perfusion within proximity of MAPOPT is associated with increased $\mathrm{PbtO}_{2}$. PRx can yield MAPOPT, lower and upper limit of autoregulation in patients following cardiac arrest. 


\section{Introduction}

The pathophysiology of hypoxic ischemic brain injury (HIBI) emanates from a primary insult during cardiac arrest and secondary injury that results partially from an inadequate balance between cerebral oxygen delivery $\left(\mathrm{CDO}_{2}\right)$ and utilization following return of spontaneous circulation (ROSC)(1). Secondary injury is increasingly recognized as a significant determinant of neurological outcome, and mitigating its deleterious effects is a mainstay of post cardiac arrest management $(1,2)$.

Following ROSC, brief hyperemia is followed by prolonged hypoperfusion, termed ' $n o$ reflow' $(1,3,4)$ which causes inadequate $\mathrm{CDO}_{2}$ and secondary injury $(1,5)$. Observational studies demonstrate that perturbations in $\mathrm{CDO}_{2}$ from hypotension $(6,7)$ and hypocapnia $(8)$ after ROSC during no-reflow are associated with worse neurological outcome. Animal models have confirmed the no-reflow phenomenon (9) and demonstrate that increasing $\mathrm{CDO}_{2}$ with mean arterial pressure (MAP) augmentation results in improved thalamic and subcortical brain tissue oxygenation $\left(\mathrm{PbtO}_{2}\right)(10,11)$. A recent systematic review suggests increased MAP is associated with improved neurologic outcome following cardiac arrest (12). Intuitively, uniform MAP augmentation seems like a plausible therapeutic intervention, however, a 'one size fits all' approach fails to account for individual physiologic differences between patients and may expose patients to adverse effects of exogenous vasopressors. Hence, recent efforts have been targeted at identifying personalized perfusion targets in HIBI (13).

Cerebral autoregulation is the inherent capacity of the cerebrovasculature to undergo cyclical cerebral vasoconstriction and vasodilation to protect the brain from ischemia and hyperemia (14), respectively. In health, cerebral autoregulation maintains stable cerebral blood flow $(\mathrm{CBF})$ over a wide range of MAP(14-16). After cardiac arrest, the preserved range of 
autoregulation is often narrowed and right shifted (17). Pressure reactivity index (PRx), a moving Pearson's correlation coefficient between MAP and intracranial pressure (ICP), can monitor autoregulation in real time and identify individualized MAP thresholds after brain injury (18). Importantly, PRx is an indirect measure of autoregulation. Fluctuations in MAP will lead to either cerebral vasoconstriction or vasodilation with resultant subsequent changes in cerebrovascular blood volume and therefore ICP. Dysfunctional autoregulation is characterized by a positive correlation between MAP and $\mathrm{CBF}$, owing to pressure passive vasodilation of the cerebrovasculature (19). Increased CBF results in increased cerebrovascular volume which itself leads to elevated ICP (20). With intact autoregulation, increasing MAP is countered by vasoconstriction, which reduces cerebrovascular blood volume and ICP, thereby producing a negative or near zero PRx (19). By plotting PRx against MAP, a U-shaped curve is formed with the nadir denoted as the optimal MAP (MAP $\left.{ }_{\mathrm{OPT}}\right)$ (21). After traumatic brain injury, perfusion within $5 \mathrm{mmHg}$ of the optimal cerebral perfusion pressure results in improved $\mathrm{PbtO}_{2}(22)$ and improved neurological outcome (21). It is unknown whether proximity to MAPOPT results in improved $\mathrm{PbtO}_{2}$ in humans with $\mathrm{HIBI}$.

As such, we conducted a prospective study of invasive neuromonitoring in patients with HIBI. The objectives of our study were: (1) characterize $\mathrm{PbtO}_{2}$ and brain hypoxia over time and its relationship to MAP, (2) characterize cerebral autoregulation as assessed by PRx over time, (3) characterize MAP ${ }_{\mathrm{OPT}}$, Lower Limit of Autoregulation (LLA) and Upper Limit of Autoregulation (ULA) over time, (4) determine the relationship between the difference in patients' actual MAP and MAPoPt (termed MAP difference $\left[\mathrm{MAP}_{\mathrm{DIFF}}\right]$ ) and $\mathrm{PbtO}_{2},(5)$ characterize the relationship between both $\mathrm{PbtO}_{2}$ and $\mathrm{PRx}$ and two potential confounders temperature and end-tidal carbon dioxide $\left(\mathrm{ETCO}_{2}\right)$. 


\section{Methods}

We conducted a prospective study in 10 patients following cardiac arrest with HIBI using multimodal neuromonitoring. The University of British Columbia Clinical Research Ethics Board approved the study (August 23, 2016 - H16-00466) and written informed consent was obtained from the temporary substitute decision maker. The study was registered at clinicaltrials.gov (NCT03609333).

\section{Study population and hospital}

The Vancouver General Hospital intensive care unit (ICU) is a quaternary closed 34 bed mixed medical-surgical unit affiliated with the University of British Columbia. The ICU provides quaternary neurotrauma care for the province of British Columbia and conducts invasive multimodal neuromonitoring in approximately 40 neurologically injured patients annually.

Patients were included if they sustained a cardiac arrest and fulfilled the following criteria: 1) time to ROSC of greater than 10 minutes with greater than 20 minutes of sustained circulation, 2) post ROSC un-confounded Glasgow coma score of less than 9, and 3) inclusion within 72 hours of cardiac arrest. We excluded patients who had any of the following criteria: 1) concurrent coagulopathy (INR $>1.5$, prothrombin time $>40$ seconds, platelet count $<100 \mathrm{x}$ $\left.10^{9} / \mathrm{L}\right)$, 2) likely cardiac catheterization within 7 days, 3) current or anticipated anticoagulant or antiplatelet therapy during the study period, 4) therapeutic hypothermia targeting a temperature under $\left.35^{\circ} \mathrm{C}, 5\right)$ prior history of severe TBI, intracranial hemorrhage or stroke, 6) anticipated withdrawal of life-sustaining therapy within 72 hours.

\section{Neurophysiologic Monitoring \& Data Collection}


ICP $\left(\right.$ Camino®, Integra Lifesciences, New Jersey, USA) and $\mathrm{PbtO}_{2}\left(\right.$ Licox ${ }^{\circledR}$, Integra Lifesciences, New Jersey, USA) catheters were inserted through a dual lumen bolt in the nondominant frontal lobe by the attending neurosurgeon or designate. We also inserted a continuous oximetric jugular venous bulb $\left(\mathrm{SjvO}_{2}\right)$ catheter (Pediasat ${ }^{\circledR}$, Edwards Lifesciences, USA). The $\mathrm{SjvO}_{2}$ catheter was placed in the dominant internal jugular vein identified by ultrasonography, and positioned at the level of the mastoid process on skull base x-ray. Near-infrared spectroscopy (NIRS) pads (INVOS®, Medtronic, Minneapolis, MN, USA) were affixed to the forehead bilaterally. The following data were recorded at $300 \mathrm{~Hz}$ using ICM+® brain monitoring software (Cambridge Enterprise, United Kingdom): ICP, MAP, $\mathrm{PbtO}_{2}, \mathrm{rSO}_{2}, \mathrm{SjvO}_{2}$, temperature, and $\mathrm{ETCO}_{2}$ ).

We also collected information relating to the cardiac arrest: etiology of the cardiac arrest, initial rhythm, whether the arrest was witnessed or unwitnessed, administration of bystander cardiopulmonary resuscitation, duration until ROSC, doses of epinephrine administered, and number of defibrillation attempts. We also collected multiple daily arterial blood gas samples.

We defined brain tissue hypoxia as a $\mathrm{PbtO}_{2}$ below $20 \mathrm{mmHg}$, as extrapolated from patients with traumatic brain injury $(23,24)$. We defined dysfunctional cerebral autoregulation as a PRx greater than $0.3(14)$. Neurologic outcomes were recorded at 6 months by telephone interview using the Glasgow Outcome Scale: (1) death, (2) persistent vegetative state, (3) severe disability - conscious but disabled, (4) moderate disability - independent for daily activities but with disabilities, and (5) good recovery - normal activities although may have minor deficits. An independent intensive care physician reviewed each subject to assess for any complications related to the invasive monitoring.

\section{Patient Management}


Our ICU uses targeted temperature management guideline for post cardiac arrest care of patients with HIBI targeting a goal temperature of $36^{\circ} \mathrm{C}$ using external surface cooling techniques and anti-pyrexial medications. Core body temperature is monitored using a continuous esophageal, rectal or urinary bladder temperature probe. Patients are sedated with intravenous propofol infusions during targeted temperature management and hemodynamic targets are set in accordance with international guidelines at the attending physician's discretion(25). All patient care decisions were left to the discretion of the attending physician and they were allowed to use the neuromonitoring data for clinical decisions as per usual practice.

Our ICU uses a tier based algorithm in patients with multimodal neuromonitoring in situ which utilizes increasing intensity of therapy aimed at keeping ICP $<25 \mathrm{mmHg}$ and with titration of MAP / CPP to target $\mathrm{PbtO}_{2}>20 \mathrm{mmHg}$ (26) using intravenous norepinephrine as the primary vasopressor. Strict adherence to arterial normoxemia $\left(\mathrm{PaO}_{2} 80\right.$ to $\left.100 \mathrm{mmHg}\right)$ was maintained for the duration of the monitoring period in each study subject to allow delineations of the physiologic relationships with $\mathrm{PbtO}_{2}$.

\section{Statistical Analysis}

A sample of 10 was chosen as the number of patients we could enroll within the time available for the study. Data collected by ICM+® brain monitoring software were exported at 10-sec averaged values into Microsoft Excel (Redmond, WA, USA). The data were then subsequently imported into Stata 15.0 (StataCorp, Texas, USA), which was used for all analyses. $\mathrm{ICM}+{ }^{\circledR}$ software calculates PRx in real-time as a moving Pearson correlation coefficient between 30 consecutive, $10 \mathrm{~s}$ averaged values of MAP and ICP signals(27). MAPOPT is determined as a secondary derivative by plotting PRx (y-axis) against the MAP range (x-axis) in 
$5 \mathrm{mmHg}$ bins. An automatic multi-window curve-fitting methodology was used to estimate MAP $_{\text {OPT }}$ (OptimalValueFlex function in ICM+). The LLA and ULA were also determined using this methodology (28), adopting a PRx optimal threshold of 0.25 . We calculated the difference

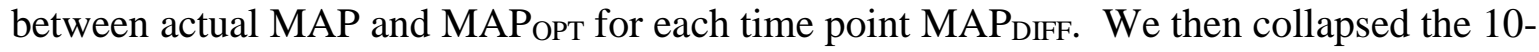
second data over 10-minutes using mean values. All analyses were two-sided and we considered $\mathrm{p}<0.05$ to be statistically significant.

Characterization of brain oxygenation and hypoxia over time and its relationship to $M A P . \mathrm{PbtO}_{2}$ and MAP were first assessed visually by plotting both over time for each individual. To characterize the degree of brain tissue hypoxia, we calculated the number of 10minute periods where $\mathrm{PbtO}_{2}$ was below $20 \mathrm{mmHg}$ for each individual. In order to estimate the average profiles of $\mathrm{PbtO}_{2}$ and MAP over time, we fitted a linear mixed model specifying 'patient' as a random-effect (STATA command $x$ treg) to account for the within-subject correlation of the data structure.

We then visually assessed the relationship of each variable of brain hypoxia $\left(\mathrm{PbtO}_{2}, \mathrm{rSO}_{2}\right.$ and $\mathrm{SjvO}_{2}$ ) by plotting each against MAP. We overlaid the scatterplot with ten locally weighted scatterplot smooths, one for each individual. For each measure of brain hypoxia $\left(\mathrm{PbtO}_{2}, \mathrm{rSO}_{2}\right.$ and $\mathrm{SjvO}_{2}$ ) we fit a linear mixed model that included MAP as a fixed effect and 'patient' as a random effect (STATA command xtreg). For modelling, we calculated the marginal and conditional $\mathrm{R}^{2}$ as a measure of the variance explained.

Characterization of cerebral autoregulation (PRx) over time. We plotted PRx over time for each individual. We determined average PRx by 10-minute periods to determine the percentage of time each patient demonstrated dysfunctional autoregulation, i.e. average PRx > 0.3 . 
Characterization of MAPOPT, LLA and ULA over time and the relationship between $M A P_{D I F F}$ and $\mathrm{PbtO}_{2}$. We first plotted MAPOPT, LLA and ULA over time. In order to link brain tissue oxygenation to cerebral autoregulation, we sought to assess the relationship between $\mathrm{PbtO}_{2}$

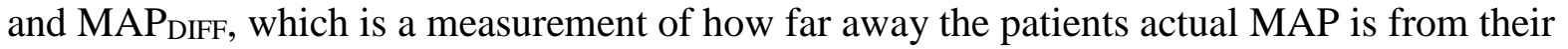
MAPOPT. One would expect that $\mathrm{PbtO}_{2}$ would be low when MAP $\mathrm{DIFF}_{\text {is }}$ iselow zero. We plotted $\mathrm{PbtO}_{2}$ vs. $\mathrm{MAP}_{\text {DIFF }}$ for each patient and overlaid the scatterplot with ten locally weighted scatterplot smooths, one for each individual. Because of the non-linear relationship between $\mathrm{PbtO}_{2}$ and $\mathrm{MAP}_{\mathrm{DIFF}}$, we modelled this relationship using fractional polynomial regression specifying 'patient' as a random-effect. To numerically describe this relationship, we calculated the mean (SD) of $\mathrm{PbtO}_{2}$ stratified by quartiles of MAPDIFF.

Characterize the relationship between both $\mathrm{PbtO}_{2}$ and $\mathrm{PRx}$ with temperature and endtidal carbon dioxide $\left(E T C O_{2}\right)$. We plotted $\mathrm{PbtO}_{2}$ and $\mathrm{PRx}$ against temperature and $\mathrm{ETCO}_{2}$ and overlaid the scatterplot with ten locally weighted scatterplot smooths for each individual. As part of an exploratory analysis, we compared PRx in survivors and non-survivors and how this changed over time. This latter analysis was performed by fitting a linear mixed model specifying 'patient' as a random-effect (STATA command xtreg) and included an interaction variable of time (hours) and favourable neurologic outcome (modeled as a dichotomous variable). 


\section{Results}

Twenty-two consecutive patients were screened between November 2016 and January 2018, of which 10 met inclusion criteria and were enrolled. Individual demographics of the patients are shown in Table 1. The median age of the cohort was 47 and seven were male. The initial rhythm was pulseless electrical activity in 9 patients and 1 patient had ventricular fibrillation. The median duration from time of cardiac arrest to insertion of multimodal neuromonitoring was 15 hours and the median duration of multimodal monitoring was 47 hours. Over the entire cohort and duration of monitoring the mean (SD) of MAP, ICP and CPP were 88 (15) $\mathrm{mmHg}, 14$ (15) $\mathrm{mmHg}$ and 75 (7) $\mathrm{mmHg}$, respectively. The mean $\mathrm{ETCO}_{2}$ was 32 (6) $\mathrm{mmHg}$ and mean core body temperature was $35.7^{\circ} \mathrm{C}(0.8)$. There were no serious adverse events related to the monitoring (intracranial hemorrhage or infection) during or after the study period.

Characterization of brain oxygenation and hypoxia over time and relationship to MAP. The mean $\mathrm{PbtO}_{2}$ in the entire cohort was 23 (8) mmHg. Of the 1944 10-minute averaged periods, $743(38 \%)$ had a $\mathrm{PbtO}_{2}$ under $20 \mathrm{mmHg}$. On an individual patient level, the mean percentage of time that patients in the cohort experienced a $\mathrm{PbtO}_{2}$ under $20 \mathrm{mmHg}$ was $38 \%$ (range 6 - 100). During the study period, the mean $\mathrm{rSO}_{2}$ was 67 (14) \% and mean $\mathrm{SjvO}_{2}$ was 74\% (11). Relationships between $\mathrm{PbtO}_{2}$ and $\mathrm{MAP}$ in individual patients over time during the monitoring period are presented in Figure 1. Both $\mathrm{PbtO}_{2}(0.20 \mathrm{mmHg}, 95 \%$ CI: $0.18-0.22)$ and MAP (0.16 mmHg, 95\% CI: $0.12-0.19)$ increased per hour during the monitoring period. Figure 2 demonstrates the relationship between MAP and indices of oxygenation. MAP was linearly related to both $\mathrm{PbtO}_{2}$ and $\mathrm{SjvO}_{2}$ but not $\mathrm{rSO}_{2}$. The large difference between the conditional and marginal $\mathrm{R}^{2}$ values observed indicates marked between-patient variability in the effects of MAP on the indices of oxygenation. 
Characterization of cerebral autoregulation (PRx) over time. The overall mean PRx was 0.23 (0.27). Out of the 2409 10-minute averaged PRx measurements, 1148 (48\%) were greater than 0.3. On an individual patient level, the median percentage of time with a PRx of greater than 0.30 was $50 \%$ (range $12-91$ ). Figure 3 presents the change of PRx over time for each patient.

Characterization of MAP $O P T, L L A$ and ULA over time and determine the relationship between $M A P_{D I F F}$ and $\mathrm{PbtO}_{2}$. The mean (SD) MAPOPT, LLA and ULA were 89 (11) mmHg, 82 (8) $\mathrm{mmHg}$ and 96 (9) $\mathrm{mmHg}$, respectively. MAPOPT, LLA and ULA over time are presented for each patient in Figure 4. The median time spent of actual MAP within $5 \mathrm{mmHg}$ of MAPOPT was $42 \%$ (range $10-78$ ). The percentage of time spent below $-5 \mathrm{mmHg}$ was $32 \%$ (range $12-74$ ) and above $+5 \mathrm{mmHg}$ from the MAPOPT was $27 \%$ (range $4-54$ ). Please see the eTable 1 for mean values of MAP, ICP, CPP, PRx, MAP ${ }_{\mathrm{OPT}}$, LLA, ULA, $\mathrm{ETCO}_{2}$ and temperature over time during the monitoring period.

In order to link brain tissue oxygenation and cerebral autoregulation, we assessed the relationship between relationship between $\mathrm{PbtO}_{2}$ and MAPDIFF (Figure 5). Fractional polynomial regression demonstrated a non-linear relationship between $\mathrm{PbtO}_{2}$ and $\left.\mathrm{MAP} \mathrm{PIFF}_{(\mathrm{p}}<0.001\right) . \mathrm{PbtO}_{2}$ increased as the difference between MAP and MAPOpt approached zero. $\mathrm{PbtO}_{2}$ leveled off as MAP $_{\text {DIFF }}$ went above zero. To numerically describe this relationship, we divided MAP DIFF $_{\text {into }}$ quartiles and calculated the associated $\mathrm{PbtO}_{2}$. Quartile $1\left(\mathrm{MAP}_{\mathrm{DIFF}} \leq-5 \mathrm{mmHg}\right)$ had a mean $\mathrm{PbtO}_{2}$ of 19 (9) $\mathrm{mmHg}$; quartile 2 (MAPDIFF -4 to $2 \mathrm{mmHg}$ ) had a mean $\mathrm{PbtO}_{2}$ of 25 (9) mmHg; quartile 3 (MAP DIFF 2 to $9 \mathrm{mmHg}$ ) had a mean $\mathrm{PbtO}_{2}$ of 23 (10) $\mathrm{mmHg}$; and quartile 4 (MAP ${ }_{\text {DIFF }}>9 \mathrm{mmHg}$ ) had a mean $\mathrm{PbtO}_{2}$ of 26 (9) $\mathrm{mmHg}$. 
Visually, there did not appear to be any relationship between either $\mathrm{PbtO}_{2}$ (eFigure 1) or PRx (eFigure 2) and temperature or $\mathrm{ETCO}_{2}$.

As part of an exploratory analysis, we compared PRx over time in survivors and nonsurvivors. The mean PRx was 0.31 (SD 0.19) in survivors and 0.16 (SD 0.32) in non-survivors. There were several patterns that emerged when visually inspecting PRx over time. Survivors appeared to either have improving (Figure 3, patients $3 \& 5$ ) or preserved (Figure 3, patients $1 \&$ 9) autoregulation. Non-survivors appeared to have dysfunctional (Figure 3, patient $2 \& 4$ ) or worsening (Figure 3, patient $4 \& 10$ ) autoregulation. In non-survivors, the PRx increased each hour by 0.0052 (95\%CI: 0.0032 to 0.0071$)$ whereas in survivors, PRx decreased over each hour by -0.0078 (95\%CI: -0.0090 to -0.0065$)$ (p-interaction <0.001). 


\section{Discussion}

We present the first prospective study using invasive multimodal neuromonitoring to evaluate brain hypoxia and cerebral autoregulation using PRx in patients with HIBI. Overall, there was a significant burden of brain hypoxia demonstrated in our cohort. Although there was a linear relationship between $\mathrm{PbtO}_{2}$ and $\mathrm{SjvO}_{2}$ with MAP, there was marked between-patient variability. We were also able to characterize cerebral autoregulation using PRx and identify MAPoPt, LLA and ULA. Interestingly, the overall mean MAPoPT was $89 \mathrm{mmHg}$ which is markedly higher than recommended by international guidelines (29). Finally, we found an association between perfusion within proximity of $\mathrm{PRx}$ derived $\mathrm{MAP}$ OPT and $\mathrm{PbtO}_{2} . \mathrm{PbtO}_{2}$ improved when the actual MAP approached within $10 \mathrm{mmHg}$ of MAPOPT, suggesting improved $\mathrm{CDO}_{2}$ within proximity to MAP OPT.

It is increasingly evident that secondary injury leads to adverse neurologic outcome and identification of ongoing brain hypoxia after ROSC is imperative. Human studies have principally focussed on establishing links between decreased $\mathrm{rSO}_{2}$ as a surrogate marker of brain oxygenation and neurological outcome, but have yielded conflicting results in its predictive utility (30-33). These conflicting results, along with technical limitations of NIRS have hindered its routine use in evaluating brain hypoxia post cardiac arrest. We present the first prospective study evaluating the burden of brain hypoxia using a direct invasive measure of cerebral oxygenation in post cardiac arrest patients with HIBI. Our results demonstrating a significant burden of brain hypoxia in our cohort during the 'no reflow' period are in keeping with prior physiologic and animal studies confirming the presence of oligemic CBF, brain hypoxia and neuronal ischemia after ROSC (29-31). Eight of ten subjects in our study exhibited an initial $\mathrm{PbtO}_{2}<20 \mathrm{mmHg}$ (figure 1) indicating that we may have captured the cerebrovascular 
physiologic profile in these subjects during 'no reflow'. Importantly, the median duration from time of cardiac arrest to data collection was 15 hours in our study, a timeline that is relatively late in the 'no reflow' period when the resolution of reduced $\mathrm{CBF}$ and $\mathrm{CDO}_{2}$ would be expected. As such, it is possible that the burden of brain hypoxia may be underestimated in our study.

Delineating the significance of brain tissue hypoxia as a modifiable factor, as opposed to an expected epiphenomenon in the natural history of HIBI, is essential. In animal models, MAP augmentation using exogenous vasopressors has demonstrated improved $\mathrm{PbtO}_{2}$ in thalamic and cortical tissue, structures which are exquisitely susceptible to secondary injury $(9,11)$. We also observed a relationship between $\mathrm{MAP}$ and $\mathrm{PbtO}_{2}$ in individual patients over time. Although significant, the variation of this relationship within patients could be explained by numerous factors including simultaneous fluctuations in other physiologic variables such as $\mathrm{ETCO}_{2}$, temperature and hemoglobin. As part of the study protocol, attending physicians were able to manipulate multiple physiologic variables to achieve $\mathrm{PbtO}_{2}>20 \mathrm{mmHg}$ and hence, we are unable to imply causality to augmented MAP and increased $\mathrm{PbtO}_{2}$. We also sought to delineate the relationships between MAP and non-invasive measures of $\mathrm{CDO}_{2}$, namely $\mathrm{rSO}_{2}$ and $\mathrm{SjvO}_{2}$. We did observe a significant association between increasing $\mathrm{MAP}$ and $\mathrm{SjvO}_{2}$, but this relationship was less robust than the relationship between $\mathrm{MAP}$ and $\mathrm{PbtO}_{2}$. As opposed to $\mathrm{PbtO}_{2}$, which is principally determined by $\mathrm{CBF}$ in the setting of stable $\mathrm{PaO}_{2}, \mathrm{SjvO}_{2}$ is additionally dependent cerebral metabolic rate and arterial oxygen content (35). These were not held constant in our study. We did not find a relationship between MAP and $\mathrm{rSO}_{2}$, raising doubts as to the validity of $\mathrm{rSO}_{2}$ by itself to monitor cerebral perfusion in HIBI. NIRS has been advocated as a surrogate measure of $\mathrm{CBF}$ and $\mathrm{CDO}_{2}$ during cardiopulmonary resuscitation and in identifying early ROSC 
(36), however, data is lacking in establishing $\mathrm{rSO}_{2}$ as a reliable estimator of $\mathrm{CBF}$ beyond the immediate post ROSC period.

Our results are consistent with epidemiologic data which suggests that post-ROSC hypotension may contribute to worse neurologic outcomes (6). A systematic review of studies examining MAP thresholds in patients following cardiac arrest demonstrated that increased MAP targets were associated with improved neurologic outcomes (12). Although generalized increased MAP targets post cardiac arrest may be a viable therapeutic intervention, the 'one size fits all' approach fails to account for physiological differences between patients. Furthermore, uniform MAP augmentation using vasopressors carries significant risks, including increased afterload on decompensated left ventricular function, arrhythmias, and mesenteric or limb ischemia. Therefore, an individualized approach to MAP targets in HIBI patients has emerged as an attractive area of research (13).

The mean MAP ${ }_{\text {OPT }}$ observed in our study $(89 \mathrm{mmHg})$ is higher than international post cardiac arrest guidelines which recommend MAP > $65 \mathrm{mmHg}$ (29). Importantly, there was considerable between and within-patient MAPOPT variability which further highlights the heterogeneous nature of HIBI. This marked heterogeneity may serve as an opportunity to therapeutically target individual MAP thresholds in patients with HIBI (13), rather than the current uniform approach. In particular, we found that cerebral autoregulation, as assessed by PRx, changes over time. Our data also suggests that the change in PRx over time may differ between survivors and non-survivors, although more data is clearly needed to elucidate this relationship. The relative importance of MAPOPT versus LLA and ULA remain unclear. It may be that LLA is a crucial physiologic parameter to target as thresholds below LLA are associated with cerebral oligemia and brain hypoxia (37). Importantly, the mean LLA (82 $\mathrm{mmHg}$ ) in our 
cohort was also still markedly higher than post cardiac arrest guidelines for MAP > $65 \mathrm{mmHg}$. The importance of LLA and ULA as perfusion targets in HIBI requires further research.

Our results are also consistent with a prior study using transcranial Doppler to assess cerebral autoregulation in patients following cardiac arrest which demonstrated a right shifted lower limit of autoregulation using transcranial Doppler (38). Furthermore, Ameloot et al. retrospectively calculated COx, a correlation coefficient between MAP and the $\mathrm{rSO}_{2}$ from NIRS to characterize autoregulation and found the mean MAРОРт in their cohort to be $85 \mathrm{mmHg}(39)$. Using a similar, but prospective COx based assessment of cerebral autoregulation, our research group recently observed a mean $\mathrm{MAP}_{\mathrm{OPT}}$ to be $76 \mathrm{mmHg}$ in a cohort of post cardiac arrest patients using COx (40).

We attempted to assess the physiologic relationship between $\mathrm{MAPOPT}_{\mathrm{O}}$ and $\mathrm{CDO}_{2}$, as measured by $\mathrm{PbtO}_{2}$. Despite heterogeneity between patients, there appeared to be a non-linear relationship between $\mathrm{MAP}$ DIFF and $\mathrm{PbtO}_{2}$. When the actual MAP was more extreme than -10 mmHg below MAPOPT, there was a linear rise of $\mathrm{PbtO}_{2}$ as $\mathrm{MAP} \mathrm{PIFF}_{\text {approached }}-10 \mathrm{mmHg}$. Between $\mathrm{MAP}_{\mathrm{DIFF}}$ of -10 to $+10 \mathrm{mmHg}$, there was a less steep rise in $\mathrm{PbtO}_{2}$, with this relationship becoming more attenuated at the top of this range. $\mathrm{MAP}_{\mathrm{DIFF}}$ above $+10 \mathrm{mmHg}$ was not associated with further improvements in $\mathrm{PbtO}_{2}$. This relationship highlights that perfusion within proximity of MAPOPT is associated with improved $\mathrm{PbtO}_{2}$ and its physiologic significance must be further evaluated. Importantly, perfusion beyond the MAP OPT was not associated with increasing $\mathrm{PbtO}_{2}$, thereby suggesting that uniformly increased MAP targets will unreliably lead to improved $\mathrm{PbtO}_{2}$ and could simply expose patients to the harmful effects of unnecessary exogenous vasopressors. Similar findings have been observed in patients with traumatic brain injury where Jaeger et al. demonstrated that $\mathrm{PbtO}_{2}$ increased significantly as actual CPP 
approached optimal CPP derived from PRx (41). These findings were further corroborated with a physiologic study revealing that perfusion pressures associated with negative PRx (intact autoregulation) were associated with improved $\mathrm{CDO}_{2}$, cerebral oxygen metabolic rate and cerebral blood flow (42). In traumatic brain injury, the clinical significance of these observed physiologic benefits are illustrated as deviation of actual CPP from the optimal CPP is associated with worse long term neurological outcomes (21). We found over half of the actual MAP measurements were more than $5 \mathrm{mmHg}$ from $\mathrm{MAP}{ }_{\mathrm{OPT}}$, suggesting an opportunity for future interventional research to target adherence to MAP ${ }_{\text {OPT. }}$

Our study is novel and characterizes the cerebrovascular pathophysiological sequelae of HIBI after cardiac arrest. Strengths of our study include the high granularity of data collection $(300 \mathrm{~Hz})$ with time-stamped neurophysiologic variables to facilitate examining the underlying relationships between various measures of brain oxygenation and $\mathrm{CDO}_{2}$. We gathered considerable longitudinal data over time with a mean duration of monitoring during the study of 46 hours and were able to evaluate the physiological profile of a key determinant of $\mathrm{CDO}_{2}$ (MAP) on brain oxygenation in HIBI. Finally, we demonstrated improved $\mathrm{PbtO}_{2}$ within proximity of MAPOPT, raising the possibility of beneficial targeted individualized perfusion targets in HIBI, however, this concept requires significant further physiologic and clinical delineation.

Our study has important limitations. Our small sample size limits our ability to draw firm conclusions on HIBI pathophysiology and indeed suggests that there are likely distinct HIBI phenotypes. This is particularly important when trying to examine for other factors that may confound the relationship between $\mathrm{MAP}$ and $\mathrm{PbtO}_{2}$, namely $\mathrm{ETCO}_{2}$ and temperature. This is the first study of its kind and necessarily is exploratory and hypothesis-generating in nature, we are 


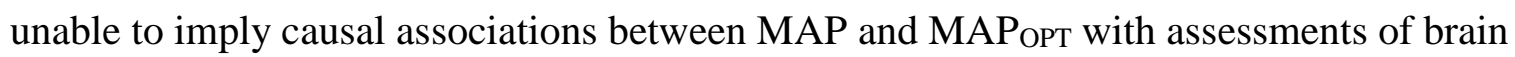
oxygenation. Instead, the observed associations in our study are intended to provide further justification to delineate their clinical and physiological significance in the future. Secondly, our definition of brain hypoxia $\left(\mathrm{PbtO}_{2}<20 \mathrm{mmHg}\right)$ is extrapolated from traumatic brain injury literature (24) and may not reflect an important threshold in patients in HIBI. Owing to the reduced cerebral metabolic demand immediately after cardiac arrest, patients with HIBI may tolerate lower levels of $\mathrm{PbtO}_{2}$ (43). Establishing $\mathrm{HIBI}$ specific $\mathrm{PbtO}_{2}$ thresholds in humans of brain hypoxia against gold standard assessments of $\mathrm{CDO}_{2}$ such as positron emission scanning is an essential gap in the current literature. In addition, the observed relationship between MAP and $\mathrm{PbtO}_{2}$ does not reflect the end organ balance of aerobic versus anaerobic metabolism.

We used PRx as a measure of autoregulation, however, we acknowledge that PRx is an indirect estimator of dynamic autoregulation which relies upon repeated longitudinal integration between fluctuations in values of MAP and changes in CBF / cerebrovascular blood volume and downstream ICP fluctuations. Dynamic changes in CBF may occur from alternative physiologic stressors independent from MAP including changes in arterial carbon dioxide tension, cerebral metabolic rate and temperature fluctuations, thereby negating the accuracy of PRx. We attempted to control these variables in our study by mandating strict $\mathrm{PaCO}_{2} 35$ to $40 \mathrm{mmHg}$, targeted temperature management $\left(36^{\circ} \mathrm{C}\right)$ and intravenous sedation using propofol to target burst suppression during the study period. However, it is possible that such goals were uniformly achieved and have affected our results.

Importantly, the patients in our cohort primarily underwent cardiac arrest from pulseless electrical activity secondary to underlying causes that likely resulted in pre-circulatory arrest cerebral ischemia (hemorrhage, hypoxia) as opposed to primary sudden cardiac arrest from 
arrythmias. As such, our results must be interpreted under premise that the majority of patients in our cohort likely fall on the severe end of the HIBI pathophysiology spectrum. Therefore, our results should not be extrapolated to patients with sudden cardiac arrest from arrythmias who undergo immediate cardiopulmonary resuscitation and further work is needed to delineate the pathophysiology in this sub-population.

Finally, increased MAP also may not translate into definitively increased CBF as we did not account for changes in cerebrovascular resistance. Future steps of study in this disease should evaluate direct measures of $\mathrm{CBF}$ as well as measures of cerebral metabolism using cerebral microdialysis and positron emission scanning.

\section{Conclusion}

Episodes of brain hypoxia are prevalent and there appears to be a linear relationship between increased MAP and $\mathrm{PbtO}_{2}$ in adult human $\mathrm{HIBI}$ patients. Perfusion within proximity of MAPOPT, as determined by $\mathrm{PRx}$, may be associated with improved $\mathrm{PbtO}_{2}$. Further physiologic research is warranted to delineate the significance of these observations. 


\section{Acknowledgements}

We would like to acknowledge the physicians and allied health staff in our intensive care unit for their support. 


\section{References}

1. Sekhon MS, Ainslie PN, Griesdale DE: Clinical pathophysiology of hypoxic ischemic brain injury after cardiac arrest: a "two-hit" model. Crit Care 2017; 21:90.

2. Busl KM, Greer DM: Hypoxic-ischemic brain injury: pathophysiology, neuropathology and mechanisms. NeuroRehabilitation 2010; 26:5-13.

3. Iordanova B, Li L, Clark RSB, et al.: Alterations in Cerebral Blood Flow after Resuscitation from Cardiac Arrest. Front Pediatr 2017; 5:174.

4. Nolan JP, Neumar RW, Adrie C, et al.: Post-cardiac arrest syndrome: epidemiology, pathophysiology, treatment, and prognostication. A Scientific Statement from the International Liaison Committee on Resuscitation; the American Heart Association Emergency Cardiovascular Care Committee; the Coun. Resuscitation 2008; 79:350-79.

5. Böttiger BW, Krumnikl JJ, Gass P, et al.: The cerebral "no-reflow" phenomenon after cardiac arrest in rats--influence of low-flow reperfusion. Resuscitation 1997; 34:79-87.

6. Trzeciak S, Jones AE, Kilgannon JH, et al.: Significance of arterial hypotension after resuscitation from cardiac arrest. Crit Care Med 2009; 37:2895-903; quiz 2904.

7. Kilgannon JH, Roberts BW, Jones AE, et al.: Arterial Blood Pressure and Neurologic Outcome After Resuscitation From Cardiac Arrest. Crit Care Med 2014; 42:2083-2091.

8. Roberts BW, Kilgannon JH, Chansky ME, et al.: Association between postresuscitation partial pressure of arterial carbon dioxide and neurological outcome in patients with postcardiac arrest syndrome. Circulation 2013; 127:2107-13.

9. Drabek T, Foley LM, Janata A, et al.: Global and regional differences in cerebral blood flow after asphyxial versus ventricular fibrillation cardiac arrest in rats using ASL-MRI. Resuscitation 2014; 85:964-971. 
10. Manole MD, Kochanek PM, Bayır H, et al.: Brain tissue oxygen monitoring identifies cortical hypoxia and thalamic hyperoxia after experimental cardiac arrest in rats. Pediatr $\operatorname{Res} 2014 ; 75: 295-301$.

11. Elmer J, Flickinger KL, Anderson MW, et al.: Effect of neuromonitor-guided titrated care on brain tissue hypoxia after opioid overdose cardiac arrest. Resuscitation 2018; 129: 121126.

12. Bhate TD, McDonald B, Sekhon MS, et al.: Association between blood pressure and outcomes in patients after cardiac arrest: A systematic review. Resuscitation 2015; 97:1-6.

13. Sekhon MS, Griesdale DE: Individualized perfusion targets in hypoxic ischemic brain injury after cardiac arrest. Crit Care 2017; 21:259.

14. Czosnyka M, Brady ÆK, Reinhard ÆM, et al.: Monitoring of Cerebrovascular Autoregulation : Facts, Myths , and Missing Links. Neurocrit Care. 2009; 10 (3):373386.

15. Tiecks FP, Lam AM, Aaslid R, et al.: Comparison of static and dynamic cerebral autoregulation measurements. Stroke 1995; 26:1014-9.

16. Czosnyka M, Miller C: Monitoring of Cerebral Autoregulation. Neurocrit Care 2014; 95102

17. Sundgreen C, Larsen FS, Herzog TM, et al.: Autoregulation of cerebral blood flow in patients resuscitated from cardiac arrest. Stroke 2001; 32:128-32.

18. Steiner L a, Czosnyka M, Piechnik SK, et al.: Continuous monitoring of cerebrovascular pressure reactivity allows determination of optimal cerebral perfusion pressure in patients with traumatic brain injury. Crit Care Med 2002; 30:733-8.

19. Sorrentino E, Diedler J, Kasprowicz M, et al.: Critical thresholds for cerebrovascular 
reactivity after traumatic brain injury. Neurocrit Care 2012; 16:258-66.

20. Czosnyka M, Smielewski P, Timofeev I, et al.: Intracranial pressure: more than a number. Neurosurg Focus 2007; 22:E10.

21. Aries MJH, Czosnyka M, Budohoski KP, et al.: Continuous determination of optimal cerebral perfusion pressure in traumatic brain injury. Crit Care Med 2012; 40:2456-63.

22. Jaeger M, Dengl M, Meixensberger J, et al.: Effects of cerebrovascular pressure reactivityguided optimization of cerebral perfusion pressure on brain tissue oxygenation after traumatic brain injury. Crit Care Med 2010; 38:1343-1347.

23. Doppenberg EM, Zauner A, Watson JC, et al.: Determination of the ischemic threshold for brain oxygen tension. Acta Neurochir Suppl 1998; 71:166-9.

24. Le Roux P, Menon DK, Citerio G, et al.: Consensus summary statement of the International Multidisciplinary Consensus Conference on Multimodality Monitoring in Neurocritical Care : a statement for healthcare professionals from the Neurocritical Care Society and the European Society of Intensive. Intensive Care Med 2014; 40:1189-209.

25. Callaway CW, Donnino MW, Fink EL, et al.: Part 8: Post-Cardiac Arrest Care.

Circulation 2015; 132:S465-S482.

26. Sekhon MS, Gooderham P, Toyota B, et al.: Implementation of Neurocritical Care Is Associated With Improved Outcomes in Traumatic Brain Injury. Can J Neurol Sci / J Can des Sci Neurol 2017; 1-8.

27. Czosnyka M, Smielewski P, Kirkpatrick P, et al.: Continuous assessment of the cerebral vasomotor reactivity in head injury. Neurosurgery 1997; 41:11-7-9.

28. Liu X, Maurits NM, Aries MJH, et al.: Monitoring of Optimal Cerebral Perfusion Pressure in Traumatic Brain Injured Patients Using a Multi-Window Weighting Algorithm. $J$ 
Neurotrauma 2017; 34

29. Peberdy MA, Callaway CW, Neumar RW, et al.: Part 9: Post-Cardiac Arrest Care: 2010 American Heart Association Guidelines for Cardiopulmonary Resuscitation and Emergency Cardiovascular Care. Circulation 2010; 122:S768-86.

30. Hayashida K, Nishiyama K, Suzuki M, et al.: Estimated cerebral oxyhemoglobin as a useful indicator of neuroprotection in patients with post-cardiac arrest syndrome: a prospective, multicenter observational study. Crit Care 2014; 18:500.

31. Nishiyama K, Ito N, Orita T, et al.: Regional cerebral oxygen saturation monitoring for predicting interventional outcomes in patients following out-of-hospital cardiac arrest of presumed cardiac cause: A prospective, observational, multicentre study. Resuscitation 2015; 96:135-41.

32. Storm C, Leithner C, Krannich A, et al.: Regional cerebral oxygen saturation after cardiac arrest in 60 patients--a prospective outcome study. Resuscitation 2014; 85:1037-41.

33. Ibrahim AW, Trammell AR, Austin H, et al.: Cerebral Oximetry as a Real-Time Monitoring Tool to Assess Quality of In-Hospital Cardiopulmonary Resuscitation and Post Cardiac Arrest Care. J Am Heart Assoc 2015; 4:e001859.

34. Manole MD, Foley LM, Hitchens TK, et al.: Magnetic Resonance Imaging Assessment of Regional Cerebral Blood Flow after Asphyxial Cardiac Arrest in Immature Rats. J Cereb Blood Flow Metab 2009; 29:197-205.

35. Rosenthal G, Hemphill JC, Sorani M, et al.: Brain tissue oxygen tension is more indicative of oxygen diffusion than oxygen delivery and metabolism in patients with traumatic brain injury. Crit Care Med 2008; 36:1917-1924.

36. Sanfilippo F, Serena G, Corredor C, et al.: Cerebral oximetry and return of spontaneous 
circulation after cardiac arrest: A systematic review and meta-analysis. Resuscitation 2015; 94:67-72.

37. Brady KM, Lee JK, Kibler KK, et al.: Continuous time-domain analysis of cerebrovascular autoregulation using near-infrared spectroscopy. Stroke 2007; 38:281825.

38. Sundgreen C, Larsen FS, Herzog TM, et al.: Autoregulation of cerebral blood flow in patients resuscitated from cardiac arrest. Stroke 2001; 32:128-32.

39. Ameloot K, Genbrugge C, Meex I, et al.: An observational near-infrared spectroscopy study on cerebral autoregulation in post-cardiac arrest patients: Time to drop "one-sizefits-all” hemodynamic targets?. Resuscitation 2015; 90:121-126.

40. Sekhon MS, Smielewski P, Bhate TD, et al.: Using the relationship between brain tissue regional saturation of oxygen and mean arterial pressure to determine the optimal mean arterial pressure in patients following cardiac arrest: A pilot proof-of-concept study. Resuscitation 2016; 106:120-125.

41. Jaeger M, Schuhmann MU, Soehle M, et al.: Continuous assessment of cerebrovascular autoregulation after traumatic brain injury using brain tissue oxygen pressure reactivity. Crit Care Med 2006; 34:1783-8.

42. Steiner LA, Coles JP, Czosnyka M, et al.: Cerebrovascular pressure reactivity is related to global cerebral oxygen metabolism after head injury. J Neurol Neurosurg Psychiatry 2003; 74:765-70.

43. Hoedemaekers CW, Ainslie PN, Hinssen S, et al.: Low cerebral blood flow after cardiac arrest is not associated with anaerobic cerebral metabolism. Resuscitation 2017; 120:4550. 


\section{Figure Legends}

Figure 1: Scatter plots and locally weighted scatterplot smoothing function of $\mathrm{PbtO}_{2}(\mathrm{mmHg}$, black) and MAP (mmHg, cyan) versus time (hours, $\mathrm{x}$-axis) for each patient. The numbers in each respective box represent the corresponding patient and demographics displayed in table 1.

Figure 2: Brain oxygen variables $\left(\mathrm{PbtO}_{2}, \mathrm{rSO}_{2}\right.$ and $\left.\mathrm{SjvO}_{2}\right)$ on the y-axis vs. mean arterial pressure (MAP) in mmHg (x axis). The dots are scatterplots between the two variables. Light grey lines are locally weighted scatterplot smoothing function for each individual patient. Black line is the overall regression line. $\mathrm{R}_{\mathrm{m}}^{2}$ is the marginal $\mathrm{R}^{2} . \mathrm{R}_{\mathrm{c}}^{2}$ is the conditional $\mathrm{R}^{2}$.

Figure 3: Scatter plots of PRx over time for all patients. The solid lines are a locally weighted scatterplot smooth for each patient. Values above the dashed line at a PRx of 0.3 represents a threshold of dysfunctional autoregulation. Patients denoted by the colour cyan were survivors. Patients in black were non-survivors.

Figure 4: Optimal MAP (MAPOPT), Lower Limit of Autoregulation (LLA) and Upper Limit of Autoregulation (ULA) over time for each patient. The solid black line is MAPOPT. The grey dashed lines represent the LLA and ULA.

Figure 5: Scatter plot between $\mathrm{PbtO}_{2}$ and the difference between MAP and MAP $\mathrm{OPT}$ (MAP $\mathrm{DIFF}_{\text {). }}$ The light grey dots are corresponding $\mathrm{PbtO}_{2}$ and MAP DIFF. The light grey solid lines are a locally weighted scatterplot smooth for each patient. The solid black line is a predicted curve generated using a restricted cubic splines model. Fractional polynomial (fp) regression confirmed there was a non-linear relationship between $\mathrm{PbtO}_{2}$ and $\mathrm{MAP}$ DIFF. The best parameterization of MAP DIFF was using $\mathrm{X}^{0.5}$ (fp 0.5).

eFigure 1: Scatter plots between $\mathrm{PbtO}_{2}$ and temperature (left panel) or ETCO2 (right panel). The light grey dots are corresponding values of $\mathrm{PbtO}_{2}$ and the variable of interest. The light grey solid lines are a locally weighted scatterplot smooth for each patient.

eFigure 2: Scatter plots between PRx and temperature (left panel) or ETCO2 (right panel). The light grey dots are corresponding values of $\mathrm{PbtO}_{2}$ and the variable of interest. The light grey solid lines are a locally weighted scatterplot smooth for each patient. Values above the dashed grey line at a PRx of 0.3 represent dysfunctional autoregulation. 